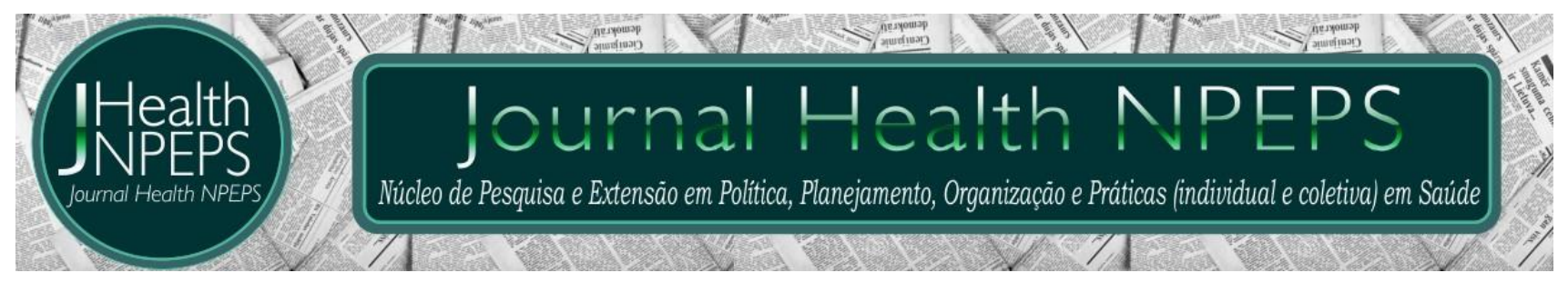

http://dx.doi.org/10.30681/252610103394

ARTIGO ORIGINAL

\title{
Perfil epidemiológico e análise de tendência do HIV/AIDS
}

\section{Epidemiological profile and trend analysis of HIV/AIDS}

Perfil epidemiológico y análisis de tendencia del HIV/SIDA

\author{
Felipe Ferraz Trindade ${ }^{1}$, Gizely Teixeira Fernandes ${ }^{2}$, Rubens Henrique Ferreira Nas- \\ cimento $^{3}$, lann Fernando Gouvêa Jabbur ${ }^{4}$, Anamaria de Souza Cardoso ${ }^{5}$
}

\section{RESUMO}

Objetivo: traçar perfil epidemiológico de HIV/AIDS em Montes Claros, Minas Gerais e analisar a linha de tendência de crescimento da doença. Método: trata-se de estudo epidemiológico, transversal, descritivo-analítico e com abordagem quantitativa. Os dados foram coletados através do DATASUS, no SINAN e SIM, referentes ao intervalo entre 1986 e 2016. A análise do modelo preditivo se deu por Regressão Linear Simples, cujos pressupostos de validação foram atendidos. Resultados: desde o primeiro diagnóstico notificado em 1986 ocorreu grande disseminação da doença, totalizando 523 casos confirmados. A distribuição foi de 59,8\% (313) para homens; 31,9\% (167) na faixa etária de 30-39 anos; 33,7\% (176) na raça parda; e 48,4\% (256) da exposição por contato sexual heterossexual. A mortalidade condiz com 71,9\% (23) do sexo masculino; 43,8\% (14) na faixa etária de 30-39 anos. A reta de tendência da epidemia denota aumento progressivo na incidência, segundo a função de $-2227,779+1,122 *(A n o)$. Conclusão: os dados avaliados permitem inferir que a epidemia de HIV/AIDS ainda se encontra em expansão. Não obstante, desde o surgimento, o perfil sociodemográfico da doença assume característica mutável, necessitando de vigilância constante para adequação de políticas de controle.

Descritores: Infecções por HIV; Epidemiologia; Modelos Lineares.

\footnotetext{
${ }^{1}$ Graduando em Medicina. Centro Universitário FIPMoc (UNIFIPMoc). Montes Claros, Minas Gerais, Brasil. E-mail: ferrazft@yahoo.com.br ORCID ID: https://orcid.org/0000-0003-0335-1167 Autor principal - Endereço para correspondência: Avenida Professora Ainda Mainartina Paraíso, número 100, apartamento 304, Ibituruna, Montes Claros, Minas Gerais, Brasil, CEP 39.408-007.

${ }^{2}$ Graduanda em Medicina. Centro Universitário FIPMoc (UNIFIPMoc). Montes Claros, Minas Gerais, Brasil. E-mail: gizely.tf@gmail.com ORCID ID: https://orcid.org/0000-0002-8121-7740

${ }^{3}$ Graduando em Medicina. Centro Universitário FIPMoc (UNIFIPMoc). Montes Claros, Minas Gerais, Brasil. E-mail: rhfnascimento@gmail.com ORCID ID: https://orcid.org/0000-0002-2344-9738

${ }^{4}$ Graduando em Medicina. Centro Universitário FIPMoc (UNIFIPMoc). Montes Claros, Minas Gerais, Brasil. E-mail: ianngouvea@yahoo.com.br ORCID ID: https://orcid.org/0000-0003-0675-6880

${ }^{5}$ Bióloga. Doutora em Ciências Biológicas. Professora dos Departamentos de Medicina e Psicologia. Centro Universitário FIPMoc (UNIFIPMoc). Montes Claros, Minas Gerais, Brasil. E-mail: anamariasc81@yahoo.com.br ORCID ID: https://orcid.org/0000-0002-5616-7286
} 


\begin{abstract}
Objective: to trace epidemiological profile of HIV I AIDS in Montes Claros, Minas Gerais and analyze the trend line of growth of the disease. Method: it is an epidemiological, cross-sectional, descriptive-analytical study with a quantitative approach. The data were collected through DATASUS, SINAN and SIM, referring to the period between 1986 and 2016. The analysis of the predictive model was by Simple Linear Regression, for which the validation assumptions were met. Results: since the first diagnosis notified in 1986, there was a great spread of the disease, totaling 523 confirmed cases. The distribution was 59.8\% (313) for men; 31.9\% (167) in the 30-39-year age group; $33.7 \%$ (176) in brown-skinned; and $48.4 \%$ (256) of heterosexual sexual contact exposure. Mortality corresponds to $71.9 \%$ (23) of males; $43.8 \%$ (14) in the age range of 30-39 years. The trend curve of the epidemic denotes a progressive increase in incidence according to the function of $-2227.779+1.122^{*}$ (year). Conclusion: the data show that the HIV I AIDS epidemic is still expanding. However, since its onset, the sociodemographic profile of the disease assumes a changing character, which requires constant vigilance for adequacy of control policies.
\end{abstract}

Descriptors: HIV Infections; Epidemiology; Linear Models.

\title{
RESUMEN
}

Objetivo: trazar perfil epidemiológico de VIH / SIDA en Montes Claros, Minas Gerais y analizar la línea de tendencia de crecimiento de la enfermedad. Método: se trata de un estudio epidemiológico, transversal, descriptivo-analítico y con abordaje cuantitativo. Los datos fueron recolectados a través de DATASUS, en el SINAN y SIM, referen- tes al intervalo entre 1986 y 2016. El análisis del modelo predictivo se dio por Regres-s Lineal Simple, cuyos supuestos de validación fueron atendidos. Resultados: desde el primer diagnóstico notificado en 1986 ocurrió una gran diseminación de la enfermedad, totalizando 523 casos confirmados. La distribución fue de 59,8\% (313) para hombres; 31,9\% (167) en el grupo de edad de 30-39 años; 33,7\% (176) en la raza parda; y 48,4\% (256) de la exposición por contacto sexual heterosexual. La mortalidad concuerda con el 71,9\% (23) del sexo masculino; 43,8\% (14) en el grupo de edad de 30-39 años. La reta de tendencia de la epidemia denota un aumento progresivo en la incidencia, según la función de -2227,779 + 1,122* (año). Conclusión: los datos evaluados permiten inferir que la epidemia de VIH / SIDA aún se encuentra en expansión. No obstante, desde el surgimiento, el perfil sociodemográfico de la enfermedad asume característica mutable, necesitando de vigilancia constante para adecuación de políticas de control.

Descriptores: Infecciones por VIH; Epidemiología; Modelos Lineales.

\section{INTRODUÇÃO}

A Síndrome da Imunodeficiência Humana Adquirida (AIDS) é uma doença causada pelo Vírus da Imunodeficiência Humana (HIV), que acomete o sistema imunológico do homem por destruir as células da série branca do sangue, res- ponsáveis pela defesa do organismo contra agentes causadores de doenças. Do ponto de vista clínico, a infecção é dividida em três fases: a fase inicial, em que a sintomatologia é muito parecida com as manifestações encontradas nas síndromes gripais; seguida de uma fase assintomática, que pode se estender por 
décadas; e a imunossupressão clinicamente relevante ${ }^{1,2}$.

O seu surgimento, nos anos de 1980, revolucionou o papel da medicina e da ciência. Evidenciou, nesse período, mudanças no sistema público de saúde, assim como melhoria no controle de qualidade dos bancos de doação de sangue, além de elevar o status da área da imunologia ${ }^{3}$.

No Brasil, os primeiros casos de morte documentados ocorreram entre os anos de 1980 e 1982, no Estado de São Paulo. No âmbito político, o país passava por um período de redemocratização pós-ditatorial, iniciado em $1964^{4,5}$. Nesse contexto, por se tratar de um momento de libertação social, o movimento gay foi fundamental no reconhecimento da AIDS como problema que necessitava de medidas governamentais específicas ${ }^{4}$, especialmente em relação aos adolescentes e jovens $^{6}$ com precocidade no contato sexual e/ou sexo desprotegido ${ }^{7}$, e consequentemente com maior vulnerabilidade para adquirir o vírus ${ }^{8,9}$.

Passados mais de 30 anos do início da epidemia, o binômio HIV/AIDS permanece como importante preocupação a nível de saúde pública global, mesmo diante dos avanços nas medidas de prevenção primária e secundária, como incentivo ao uso de preservativos, criação dos Centros de Testagem e Aconselhamento (CTA) para diagnóstico precoce da infecção, e fornecimento gratuito da terapia antirretroviral. Talvez, um dos desafios atuais colocados aos profissionais de saúde e governo, seja a falta de compreensão sobre particularidades epidemiológicas da doença, que analisadas de forma global não conseguem direcionar intervenções locais. Diante disso, o estudo objetivou traçar perfil epidemiológico de HIV/AIDS em Montes Claros (MG) e analisar a linha de tendência de crescimento da doença.

\section{MÉTODO}

Trata-se de um estudo epidemiológico, transversal, descritivo-analítico e com abordagem quantitativa. Os dados foram obtidos pela plataforma online do DATASUS durante o segundo semestre do ano de 2018, nos bancos de dados SINAN (Sistema de Informações de Agravos de Notificação) e SIM (Sistema de Informação sobre Mortalidade), tabulados pelo recurso TabWin. Para fins de comparação fidedigna, foram agrupadas faixas etárias em intervalos que permitissem a comparação entre os bancos citados, uma vez que a segmentação proposta por ambos é divergente. 
A população estudada abarcou indivíduos de ambos os sexos, diagnosticados com AIDS/HIV, no município de Montes Claros - MG, entre janeiro de 1986 e dezembro de 2016, analisados sob as variáveis de sexo, etnia/raça, escolaridade, modalidade de exposição, faixa etária, distribuição temporal e número de óbitos.

A escolha do período inicial analisado se baseou nos primeiros casos notificados no sistema DATASUS em Montes Claros-MG, e o término do intervalo em 2016 se deu por conta de os dados do ano seguinte ainda não estarem disponíveis por completo. Não obstante, o período eleito representa desde o início da disseminação até os diagnósticos atuais, permitindo analisar por completo a evolução da doença ${ }^{10}$.

Quanto ao número de óbitos, o período analisado é de 1992 a 2016. O espaço temporal é distinto do período utilizado para prevalência devido à disponibilidade dos dados no portal DATASUS. Os dados referentes a mortalidade por HIV/AIDS em Montes Claros-MG se iniciam no ano de 1992, impossibilitando fazer uma comparação com a prevalência nos primeiros anos em que a doença começou a ser notificada.

Para analisar a significância dos dados descritos, foi adotado o teste Qui- quadrado de Pearson, que testa a hipótese nula afirmando-se que a distribuição de um evento observado é consistente com uma distribuição teórica particular, com resultados de $p<0,05$.

Dentre os modelos preditivos disponíveis para a análise da dispersão dos casos em séries históricas, elegeu-se a regressão linear simples, método bivariado que constrói uma linha reta entre as duas variáveis, em que a soma das distâncias quadradas entre os pontos é mínima. Nesse instrumento, adotou-se como variável dependente $(\mathrm{Y})$ o número de casos de HIV diagnosticados em Montes Claros, e a variável independente $(X)$ o ano de diagnóstico. Para análise dos dados, foi utilizado o programa estatístico Statical Package for Social Science (SPSS).

Em respeito ao rigor estatístico, foram verificados os pressupostos básicos de validação da regressão linear, sendo eles: intervalo de confiança, com $n=31$ na variável independente; independência, por meio do impedimento de duplicidade pela tabulação anual; linearidade, com teste de Correlação de Pearson resultando em 0,865; multicolinearidade comprovadamente ausente pelo teste de Durbin-Watson com resultado de 1,457; análise de Resíduos com ausência de outliers pelo teste de Outliers com interva- 
lo resultante $-2,522$ e $+2,282$; normalidade de Resíduos afirmada pelo teste de Shapiro-Wilks; e homocedasticidade testada pela função de White.

\section{RESULTADOS E DISCUSSÃO}

O número total de casos notificados no período analisado foi de 523, sendo $59,8 \%$ (313) no sexo masculino. Esses dados são semelhantes quando comparados aos dados nacionais, os quais registraram $67,9 \%$ (131.969) dos casos no sexo masculino, expressando uma razão homem/mulher de 25:10, no período entre 2007 e junho de $2017^{11}$.

0 conhecimento da maior prevalência da doença no sexo masculino é importante para direcionar as políticas públicas, e dar maior enfoque à saúde do homem. Para isso, ações de sensibilização e conscientização vêm sendo feitas, particularmente quanto ao uso do preservativo masculino ${ }^{12}$.

A faixa etária com maior prevalência de diagnóstico por HIV/AIDS foi entre 30 a 39 anos, com 31,9\% (167) dos casos notificados. Em contraponto, a faixa etária menos acometida, foi em maiores de 60 anos, com 2,5\% (13). É importante destacar a representatividade de casos em menores de 29 anos, com $31,5 \%$ (165) dos casos notificados.
Em um estudo realizado em Caxias (MA), no período de 1989 a agosto de 2016, identificou-se quantitativos próximos à realidade de Montes Claros (MG), tanto em faixa etária quanto ao perfil dos acometidos ${ }^{13}$. Ressalta-se, entretanto, a ausência de dados em relação a faixa etária e a modalidade de exposição ${ }^{12}$, que poderiam oferecer maior entendimento sobre as práticas e comportamentos de risco.

Apesar de a menor prevalência estar na faixa etária acima dos 60 anos, está havendo um crescente aumento de casos de HIV/AIDS entre idosos. Com frequência, essa população é rotulada como um segmento populacional indiferente às práticas sexuais. No entanto, o maior acesso aos medicamentos para distúrbios eréteis tem contribuído para desmistificação da hipoatividade sexual, bem como para o aumento da transmissão de Infecções Sexualmente Transmissíveis (IST) nessa fração ${ }^{14}$.

Ademais, as alterações fisiológicas do envelhecimento determinam uma menor capacidade do sistema imunológico do idoso, tornando-o mais suscetível a infecções e menos responsivo contra os agentes agressores. Não obstante, sintomas inespecíficos como astenia e anorexia, comuns nesse ciclo de vida, difi- 
cultam 0 diagnóstico precoce de HIV/AIDS, logo retarda o tratamento ${ }^{14}$. Em relação à etnia/raça, no período analisado, a maior prevalência ocorreu entre pardos, com 33,4\% (176) dos casos notificados. Todavia, há uma porcentagem significativa de notificações em que esse item foi ignorado, representando um total de $46,8 \%$ (245), fato que se repetiu na variável de escolaridade. Isso implica em uma crítica à forma de registro e alimentação dos bancos e sistemas de informação. Devido a isso, os dados públicos disponibilizados, normalmente não expressam com fidedignidade alguns indicadores, já que os aspectos relativos a determinadas doenças e ocorrências são incompletos ou subnotificados.

Quando comparado a outro estudo realizado no norte de Minas Gerais, no período de janeiro de 1987 a julho de 2007, a análise étnica/racial indica que a maior prevalência também ocorre entre pardos, representando $77,89 \%$ (356) do total de casos. Contudo, a porcentagem de ignorados não é significativa, com apenas $1,96 \%(9)^{15}$.

Em relação a escolaridade, o perfil mais acometido foi entre indivíduos com 4 a 8 anos de estudo, somando 20,2\% (106), seguido por 9 e 11 anos de estudo com $15,1 \%$ (79) dos casos. Seme-
Ihante a esses dados, em Caxias (MA) há uma maior prevalência da HIV/AIDS em indivíduos com menos de 7 anos de escolaridade. Entretanto, a porcentagem de ignorados no perfil escolar não é tão abrangente ${ }^{13}$.

Sabe-se, que no Brasil, a maior prevalência do HIV/AIDS está diretamente relacionada à baixa instrução e a vulnerabilidade econômica. Em contrapartida, quanto maior a escolaridade, maior o estímulo e acesso a conhecimentos sobre riscos à saúde e transmissibilidade de doenças ${ }^{16}$.

A modalidade de exposição mais acometida no período analisado foi por relação sexual, do tipo heterossexual, com total de 253 casos. Em menor número, os usuários de drogas injetáveis com 4,4\% (23) e por transmissão vertical com 2,5\% (13). Comparativamente, entre os anos de 1986-1989, a maior prevalência de infecção era de homossexual/bissexual com 52,2\%. Já no período entre 1993-1996, a maior prevalência se deu na modalidade heterossexual com $25,9 \%$, seguido pelos usuários de drogas injetáveis com 24,4\%10.

As modalidades de exposição relacionadas à infecção do HIV/AIDS no Brasil sofreram grandes variações no decorrer do tempo em relação à prevalência. Nos primeiros anos de epidemia, a 
modalidade mais comum era a transmissão sexual acometendo principalmente o grupo de homens que fazem sexo com homens $(\mathrm{HSH})^{12}$.

Tabela 1 - Casos notificados de HIV/AIDS. Período de 1986 a 2016. Montes Claros - MG, Brasil.
Variáveis

Sexo

Masculino

Idade

Abaixo de 29

anos

De 30 a 39

anos

De 40 a 49

anos

De 50 a 59

anos

Acima de 60

anos

Raça

Branca

Preta

Parda

Outros

Ignorado

Escolaridade

Nenhuma

De 1 a 3 anos

De 4 a 8 anos

De 9 a 11

anos

Acima de 11

anos

Ignorado

Exposição

Homossexual

Bissexual

Heterossexual

UDI

Transfusão

Transmissão

Vertical

Ignorado

Fonte: DATASUS n

Casos

$\% \quad$ Valor de

$p$

0,000

$40,2 \%$

$59,8 \%$

0,000

$31,5 \%$

$31,9 \%$

$24,1 \%$

$9,9 \%$

$2,5 \%$

0,000

$61 \quad 11,7 \%$

$31 \quad 5,9 \%$

$176 \quad 33,7 \%$

$10 \quad 1,9 \%$

$245 \quad 46,8 \%$

0,000

$2,2 \%$

$6,8 \%$

$20,2 \%$

$15,1 \%$

$6,5 \%$

$48,9 \%$

0,000

$10,5 \%$

$5,4 \%$

$48,4 \%$

$4,4 \%$

$1,0 \%$

$13 \quad 2,5 \%$

$146 \quad 27,9 \%$
Atualmente, a transmissão heterossexual é a mais prevalente, o que contribui também para um aumento na prevalência no sexo feminino, processo que foi denominado de "heterossexualização" e "feminização"10.

A maior taxa de mortalidade ocorre também no público masculino, ratificando uma necessidade de maior atenção em relação às estratégias governamentais para essa clientela, que apesar de existir políticas pública voltada à saúde do homem, pouco se vislumbra de mudanças no cotidiano assistencial dos serviços de saúde. Em relação a faixa etária com maior porcentagem de mortalidade foi de 30 a 39 anos, com $43,8 \%$ (14) de óbitos notificados. No Brasil, esta faixa etária também representou o grupo de maior mortalidade na década de $80(38,1 \%)$ e na década de 90 $(40,2 \%)^{17}$, mas com os regimes terapêuticos atuais, a tendência é que haja redução da morbimortalidade relacionada à doença ${ }^{18}$.

A etnia mais acometida em relação à mortalidade também é a parda, com 28,1\% (9) dos óbitos. Assim como a prevalência, os dados de mortalidade a respeito da etnia/raça representam uma maioria de ignorados. Em estudo realizado em Porto Alegre (RS), a etnia com maior taxa de mortalidade foi a preta ${ }^{19}$. 
Em relação a escolaridade nos casos de óbitos, o registro como ignorado representa uma elevada proporção em relação aos outros grupos analisados. Porém, vale ressaltar que assim como a prevalência, a mortalidade nos indivíduos com baixo grau de escolaridade representa significativa parcela do total de casos.

Quanto à variável do estado civil, o grupo com maior mortalidade é dos solteiros, representando $65,7 \%$ (21) do total de óbitos notificados, seguido pelos casados com um total de $12,5 \%$ (4). Assim como no Brasil, o estado civil com maior taxa de óbitos na década de 1990 é a de solteiros, representando $66,2 \%$ das mortes ${ }^{17}$.

A maior porcentagem no grupo de solteiros implica em questões relacionadas aos comportamentos de risco. Os solteiros são um grupo mais propenso a contaminação e transmissão das IST assim como das coinfecções devido a maior promiscuidade, e falta de cuidados com a saúde, de modo que têm que cuidar de si mesmos sozinhos ${ }^{20,21}$.

Em relação a Figura 1, a reta de tendência da epidemia do número de casos de HIV/AIDS no município de Montes Claros-MG, entre os anos de 1986 a 2016 denota aumento progressivo na incidência com o decorrer do período ana- lisado. Com base na observação dessa regressão, é possível prever o quantitativo de pessoas acometidas no decorrer dos anos, de acordo com: $[F(1,29)=$ 85,808, $\mathrm{p}<0,001 ; \mathrm{R} 2=0,747]$. Diante disso, o crescimento do número de casos de HIV pode ser previsto, utilizando-se a seguinte fórmula: $-2227,779+$ $1,122^{*}$ (Ano).

Tabela 2 - Dados de mortalidade por HIV/AIDS. Período de 1986 a 2016. Montes Claros - MG, Brasil. Variáveis

\begin{tabular}{|c|c|c|c|}
\hline Sexo & $\mathrm{n}$ & $\%$ & $\begin{array}{c}\text { Valor de } p \\
0,013\end{array}$ \\
\hline Feminino & 9 & $28,1 \%$ & \\
\hline Masculino & 23 & $71,9 \%$ & \\
\hline Idade & & & 0,007 \\
\hline $\begin{array}{l}\text { Abaixo de } 29 \\
\text { anos }\end{array}$ & 5 & $15,6 \%$ & \\
\hline De 30 a 39 anos & 14 & $43,8 \%$ & \\
\hline De 40 a 49 anos & 6 & $18,8 \%$ & \\
\hline De 50 a 59 anos & 6 & $18,8 \%$ & \\
\hline $\begin{array}{l}\text { Acima de } 60 \\
\text { anos }\end{array}$ & 1 & $3,0 \%$ & \\
\hline Raça & & & 0,000 \\
\hline Branca & 1 & $3,1 \%$ & \\
\hline Preta & 1 & $3,1 \%$ & \\
\hline Parda & 9 & $28,1 \%$ & \\
\hline Outros & 0 & $0 \%$ & \\
\hline Ignorado & 21 & $65,7 \%$ & \\
\hline Escolaridade & & & 0,000 \\
\hline Nenhuma & 2 & $6,3 \%$ & \\
\hline De 1 a 3 anos & 2 & $6,3 \%$ & \\
\hline De 4 a 8 anos & 8 & $25,0 \%$ & \\
\hline De 9 a 11 anos & 1 & $3,1 \%$ & \\
\hline $\begin{array}{l}\text { Acima de } 11 \\
\text { anos }\end{array}$ & 0 & $0 \%$ & \\
\hline Ignorado & 19 & $59,3 \%$ & \\
\hline Estado Civil & & & 0,000 \\
\hline Solteiro & 21 & $65,7 \%$ & \\
\hline Casado & 4 & $12,5 \%$ & \\
\hline Viúvo & 3 & $9,4 \%$ & \\
\hline Separado & 3 & $9,4 \%$ & \\
\hline Ignorado & 1 & $3,0 \%$ & \\
\hline
\end{tabular}

Fonte: DATASUS. 
Estima-se, portanto, que ao longo dos próximos 30 anos, caso não seja realizada nenhuma intervenção profilática, o aumento do número de casos poderá ser duplicado, de 34 casos (ano de 2016) para 68 (em 2046).

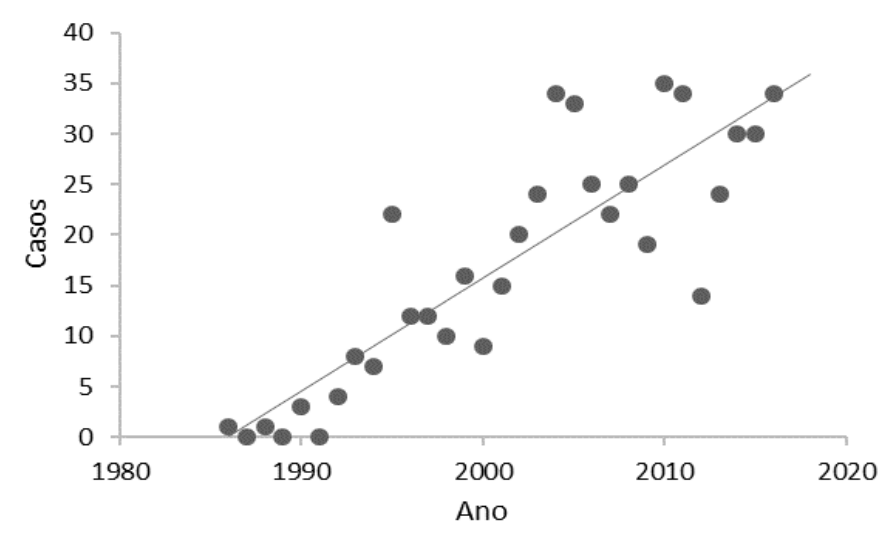

Figura 1 - Casos de HIVIAIDS e reta de tendência. Período de 1986 a 2016. Montes Claros MG, Brasil.

Os dados brasileiros atuais demonstram que a AIDS, ao contrário do que é observado em nível mundial, atingiu seus piores indicadores desde o início da doença, mesmo diante da maior conscientização quanto à prática sexual segura e aspectos relacionados com a comorbidade de pessoas que vivem com a doença. Esse crescimento ocorre principalmente entre os homossexuais, indivíduos do sexo masculino e moradores de centros urbanos, devido, sobretudo, à diminuição da transmissão em outros grupos específicos como: usuários de drogas injetáveis e pessoas heterossexuais ${ }^{22}$.

O Ministério da Saúde aponta que o número de pessoas infectadas que conhecem o seu diagnóstico, mas que não são acompanhadas por um serviço de saúde pode ser representado por 296.000 e, portanto, são considerados como importantes fontes de infecção ${ }^{8}$.

0 número de casos entre mulheres sempre foi inferior ao dos homens ao longo do período analisado. Sendo que, dos 523 casos selecionados para o estudo, 40,2\% (210) correspondem às mulheres (Figura 2 - A e B). As linhas de tendências nesses dois grupos mostraram crescimento constante em ambos os sexos. Os modelos foram estatisticamente significativos, com $\mathrm{R}^{2}$ de $62,4 \%$ para os casos de Homens [número de casos de HIV previsto, corresponde a -2227,779 + $1,122^{*}$ (Ano)], e $47,7 \%$ para as mulheres [número de casos de HIV previsto, corresponde a $-947,735+0,477^{*}($ Ano $\left.)\right]$.

A

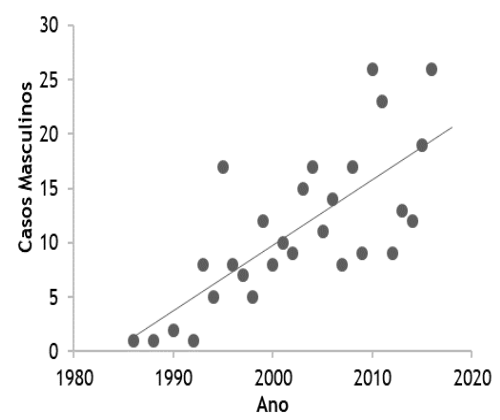

Figura 2. Gráfico de dispersão dos casos e regressão linear, com reta de tendência do sexo masculino (Painel A) e sexo feminino (Painel B) 
A avaliação da infecção de acordo com a variável sexo é complexa visto que padrões culturais e sociais também definem os papéis sexuais que homens e mulheres desempenham na sociedade. Dessa forma, apesar do número de mulheres infectadas ter aumentado nas últimas décadas, observa-se ainda, desproporção da razão masculino/feminino, com maior expressividade entre homens.

Tal fato é explicado devido à baixa conscientização dos homens acerca da importância em acessar os serviços de prevenção e assistência. Não obstante, o homem tende a considerar-se "invulnerável", rejeitando a possibilidade de adoecer, consequentemente deixando-o susceptível ao adoecimento ${ }^{8,23}$.

\section{CONCLUSÃO}

Os dados avaliados no período entre 1986 e 2016 na cidade de Montes Claros - MG tornaram possível inferir que a epidemia de HIV/AIDS ainda se encontra ativa, apesar das mudanças sociais.

A feminização do processo de doença foi evidenciada pelo aumento do número de mulheres infectadas, bem como pela diminuição da razão masculino/feminino nas últimas décadas. 0 expressivo aumento entre os jovens (especialmente aqueles com menos de 7 anos de escolaridade) e entre os idosos, demonstrou obsolescência da premissa de não susceptibilidade desses grupos à IST.

A prevalência ainda majoritária no sexo masculino reforça a difusão da autoconvicção de invulnerabilidade masculina, o que somado à maior negligência quanto ao autocuidado acaba por aumentar, indiretamente, a mortalidade masculina e o número de mulheres infectadas.

Quanto às limitações do presente estudo, aponta-se que o uso de dados secundários não permite controlar potenciais erros de registro ou sequer o preenchimento pleno das variáveis em apreciação, fato que se apresentou como principal contrariedade à análise integral da amostra. No tocante a subnotificação, também usual nessa prática de coleta, supõe-se irrelevância no presente estudo por se tratar de um agravo de notificação compulsória, cuja terapêutica só é acessível após registro do caso. Dessa forma, acredita-se que, a despeito das limitações expostas, os resultados apresentados aduzam confiabilidade.

Diante do exposto, conclui-se que há necessidade de implantação de políticas públicas abrangentes, visto que a mudança do perfil sociodemográfico da doença não mais isenta nenhum segmento populacional. A discussão acerca das 
formas de transmissão e da gravidade da doença é de suma importância para que haja conscientização quanto ao uso de preservativo, bem como de outros métodos, uma vez que o contato sexual é a principal forma de disseminação.

Por fim, é fundamental que haja conscientização dos profissionais de saúde quanto à demanda por preenchimento adequado das fichas de notificação, uma vez que essa é uma das mais relevantes ferramentas de estudo epidemiológico nacional, atuando, secundariamente, como guia para formulação de políticas públicas.

\section{REFERÊNCIAS}

1. Neves JAC, Melo NS, Souza JC, Oliveira MM, Cerqueira TF. Processo saúde-doença: a sexualidade e a AIDS na terceira idade. Enferm rev. 2015; 18(1):121-135.

2. EUA. Department of Health \& Human Services. Symptoms of HIV. Maio de 2017 [acesso em 2018 mai 12]. Disponível em:

https://www.hiv.gov/hivbasics/overview/about-hiv-andaids/symptoms-of-hiv.

3. Barata GF. A primeira década da Aids no Brasil: o Fantástico apresenta a doença ao público (1983 a 1992). Dissertação [Mestrado em História]. Journal Health NPEPS. 2019 jan-jun; 4(1):153-165
Universidade de São Paulo: Faculdade de Filosofia, Letras e Ciências Humanas, 2006 [acesso em 2018 mar 8].

Disponível em: http: / /www.teses.usp.br/teses/disp oniveis/8/8138/tde-07072006124258/pt-br.php.

4. Barros SG, Vieira-da-Silva LM. A gênese da política de luta contra a aids e o Es- paço Aids no Brasil (19811989). Rev saúde pública. 2016; 50:43.

5. Waldvogel B, Morais LCC. Mortalidade por Aids em São Paulo: dezoito anos de história. In: Anais Encontro Nacional de Estudos Populacionais da ABEP; 2016. p. 2131- 2145. [acesso em 2018 mai 09]. Disponível em: http://www.abep.org.br/publicacoe s/index.php/anais/article/download 1925/890.

6. Soares JP, Silva ACO, Silva DM, Freire MEM, Nogueira JA. Prevalência de fatores de risco para o HIV/AIDS em populações vulneráveis: uma revisão de literatura. Arq catarin med. 2017; 46(4):182-194.

7. Gúercio PMS. História da Aids no Brasil. Departamento de Epidemiologia em Serviços de saúde, 2008 [acesso em 2018 mar 08]. Disponível em: https://www.pjf.mg.gov.br/secretar ias/ss/aids_dst/arquivos/historia_br asil.pdf 
8. Brasil, Ministério da Saúde. Boletim Epidemiológico - HIV/aids - 2015. Brasília, DF: 2014 [acesso em 2018 mar 08]. Disponível em: http: / / www.aids.gov.br/pt$\mathrm{br} / \mathrm{pub} / 2015 /$ boletimepidemiologico-hivaids-2015.

9. Fonseca MGP, Barreira D. A evolução da mortalidade por AIDS no país, segundo sua distribuição geográfica. In: Boletim Epidemiológico aids; 2006 [acesso em 2018 mar 08]. Disponível em: http://bvsms.saude.gov.br/bvs/peri odicos/Boletim_jul_set_2000.pdf.

10. Szwarcwald C, Bastos F, Esteves M, Andrade C. A disseminação da epidemia da AIDS no Brasil, no período de 1987-1996: uma análise espacial. Cad saúde pública. 2000; 16(suppl 1):S07-S19.

11. Brasil, Ministério da Saúde, Secretaria de Vigilância em Saúde. Boletim epidemioló- gico HIV/Aids 2017. 2017;20(1). [acesso em 2018 mar 08]. Disponível em: http: / /www.aids.gov.br/pt$\mathrm{br} / \mathrm{pub} / 2017 /$ boletimepidemiologico-hivaids-2017

12. Brito A, Castilho E, Szwarcwald C. AIDS e infecção pelo HIV no Brasil: uma epidemia multifacetada. Rev Soc Bras Med Trop. 2001; 34(2):207217.
13. Abreu SR, Pereira BM, Silva NM, Moura LRP, Brito CMS, Câmara JT. Estudo epidemiológico de pacientes com infecção pelo vírus da imunodeficiência humana/síndrome da imunodeficiência adquirida (hiv/aids), Caxias-MA. Rev Interdiscip. 2016; 9(4):132-141.

14. Santos A, Assis M. Vulnerabilidade das idosas ao HIV/AIDS: despertar das políticas públicas e profissionais de saúde no contexto da atenção integral: revisão de literatura. Rev bras geriatr gerontol. 2011; 14(1):147-157.

15. Pereira JA, Marques RH, Fonseca LVL, Eleutério AM, Bonfim MLC, Dias OV. Infecção Pelo HIV e AIDS em Município do Norte de Minas Gerais. Rev APS. 2011; 14(1):39-49.

16. Gomes R, Ceccato M, Kerr L, Guimarães M. Fatores associados ao baixo conhecimento sobre HIV/AIDS entre homens que fazem sexo com homens no Brasil. Cad saúde pública. 2017 [acesso em 2018 mar 8]; 33(10):e00125515.

17. Reis A, Santos E, Cruz M. A mortalidade por aids no Brasil: um estudo exploratório de sua evolução temporal. Epidemiol serv saúde. 2007;16(3):195-205.

18. Bonolo P, Gomes R, Guimarães M. Adesão à terapia anti-retroviral 
(HIV/aids): fatores associados e medidas da adesão. Epidemiol serv saúde. 2007; 16(4):261-278.

19. Cunha A, Cruz M, Torres R, Cunha A, Cruz M, Torres R. Tendência da mortalidade por aids segundo características sociodemográficas no Rio Grande do Sul e em Porto Alegre: 2000-2011. Epidemiol serv saúde. 2016; 25(3):477-486.

20. Costa D, Zago M, Medeiros M. Experiência da adesão ao tratamento entre mulheres com Vírus da Imunodeficiência Humana/Síndrome da Imunodeficiência Adquirida. Acta paul enferm. 2009; 22(5):631-637.
21. Barbosa I, Costa Í. Estudo Epidemiológico da Coinfecção TuberculoseHIV no Nordeste do Brasil. Rev patol trop. 2014; 43(1):27-38.

22. Grangeiro A, Castanheira E, Nemes M. A re-emergência da epidemia de aids no Brasil: desafios e perspectivas para o seu enfrentamento. Interface (Botucatu). 2015; 19(52):58.

23. Amaral RS, Carvalho STRF, Silva FMAM, Dias RS. Soropositividade para HIV/AIDS e características sociocomportamentais em adolescentes e adultos jovens. Rev pesq saúde. 2017; 18(2):108-113.

Conflito de interesses: Os autores declaram não haver conflito de interesses.

\section{Participação dos autores:}

- Concepção: Trindade FF, Fernandes GT, Nascimento RHF, Jabbur IFG, Cardoso AS.

- Desenvolvimento: Trindade FF, Fernandes GT, Nascimento RHF, Jabbur IFG, Cardoso AS.

- Redação e revisão: Trindade FF, Fernandes GT, Nascimento RHF, Jabbur IFG, Cardoso AS.

Como citar este artigo: Trindade FF, Fernandes GT, Nascimento RHF, Jabbur IFG, Cardoso AS. Perfil epidemiológico e análise de tendência do HIV/AIDS. J Health NPEPS. 2019; 4(1):153-165. 